\title{
MODERNIZACIÓN DE EQUIPO FEEDBACK MS-150 PARA EL APRENDIZAJE ACTIVO EN INGENIERÍA DE CONTROL
}

\author{
Perfecto Reguera Acevedo \\ Universidad de León, diepra@unileon.es \\ Miguel Ángel Prada Medrano \\ Universidad de León, mapram@unileon.es \\ Antonio Morán Álvarez \\ Universidad de León, a.moran@unileon.es \\ Juan José Fuertes Martínez \\ Universidad de León, diejfu@unileon.es \\ Manuel Domínguez González \\ Universidad de León, diemdg@unileon.es \\ Serafín Alonso Castro \\ Universidad de León, saloc@unileon.es
}

\begin{abstract}
Resumen
En este artículo se presenta el desarrollo $e$ integración de elementos hardware para construir un sistema de adquisición de datos que permite modernizar el equipo didáctico Feedback MS-150 con el fin de ampliar su tiempo de vida y aumentar el alcance de los conceptos de control que se pueden explicar con él. El sistema de adquisición no supera los $100 €$
\end{abstract}

Palabras Clave: adquisición de datos, arduino, simulink, I2C, Feedback MS-150.

\section{INTRODUCCIÓN}

En el campo de la enseñanza de control, durante los últimos años, se ha prestado gran interés a diseñar, implementar y utilizar estructuras tecnológicas (laboratorios remotos) que permitan el acceso, vía internet, a equipos remotos con el fin de que los alumnos puedan realizar ensayos y prácticas no presenciales [4].

En la Universidad de León, dentro de los equipos remotos que se ofrecen a los alumnos para utilizarlos, disponemos de un equipo Feedback MS-150. Este equipo es muy popular en todas las universidades del país por su robustez, flexibilidad y la calidad pedagógica de las prácticas que con él se pueden plantear.
Aunque los laboratorios remotos presentan grandes ventajas [5], hay que tener en cuenta que ofrecer acceso remoto a un equipo supone hipotecar el uso de dicho equipo para que sólo se haga de forma "no presencial" y además no hay que desdeñar lo que es la práctica presencial, en la que el alumno manipula (realiza cableado), toca, ve y realiza el trabajo directamente, sin intermediarios. La "presencia" supone un plus en su educación difícil de ser sustituido.

Cuando, como docentes, intentamos acercar a los alumnos del primer curso de ingeniería de control los conceptos de regulador, acción de control, sistema, captador, actuador, ...; el alumno debe poder "sentirlos", "verlos", "tocarlos". Es en este curso introductorio donde hay que hacer más hincapié en las prácticas de tipo presencial. Las prácticas en laboratorio (aprender con lo que se hace) contribuyen al aprendizaje activo de los alumnos puesto que hacen uso de algunos de los 7 principios de buenas prácticas en educación [1][2]:

1. Comunicación bidireccional alumnodocente. La posibilidad de que los alumnos puedan recurrir a alguien cuando se "atasquen", que el docente aclare un poco el bosque o centre los objetivos es de gran valor educativo para los alumnos.

2. Reciprocidad y cooperación entre estudiantes. Buen aprendizaje es un esfuerzo colaborativo y social, no competitivo ni aislado [6]. Las prácticas en grupo de no 
más de 3 personas son una herramienta muy útil.

3. Aprendizaje activo. Las personas recuerdan sólo el $20 \%$ de lo que oyen y el $90 \%$ de lo que dicen y hacen por sí mismos [3].

4. Retroalimentación inmediata. Los alumnos deben ser capaces de conocer lo que saben y lo que no saben para que puedan focalizar sus esfuerzos en la dirección adecuada. Las prácticas constituyen una forma de autoevaluación del conocimiento que se posee de la materia.

5. Tiempo efectivo en la tarea. Las prácticas "entrenan" a los alumnos en cómo gestionar de forma eficiente el tiempo que deben dedicar a determinadas tareas.

6. Altas expectativas. Los docentes tienen que esperar lo mejor de sus alumnos y proponer prácticas de gran calidad. Esta idea es una profecía que se cumple cuando docentes e instituciones hacen lo posible y lo imposible por conseguir que los alumnos alcancen su completo potencial.

7. Respeto a la diversidad del talento y distintos modos de aprendizaje. Los alumnos necesitan oportunidades para mostrar su talento, por lo que ofrecer distintas posibilidades de aprendizaje que sean útiles para ellos es imperativo: prácticas en grupo, autoevaluación mediante problemas prácticos propuestos, simulaciones, etc.

En la Universidad de León, sólo disponemos de 4 equipos Feedback MS-150 para impartir contenidos de tipo práctico (grupos B3) en las asignaturas de ingeniería de control (asignaturas de Ingeniería de Control y Sistemas de Control de las titulaciones de los grados de Electrónica y Automática, Ingeniería Aeroespacial e Ingeniería Eléctrica). Por tanto, necesitamos tener la posibilidad de poder conectar/desconectar de una forma sencilla dichos equipos de lo que sería la infraestructura de laboratorio remoto y además ofrecer una modernización de dichos equipos que permita realizar prácticas de un mayor valor añadido sin un desembolso de coste adicional exagerado.

La modernización consiste en la creación de un hardware específico (para el Feedback) de adquisición de datos y la utilización de unas tarjetas E/S de bajo coste que permitan comunicación por I2C. La idea es realizar un diseño general que podría ser utilizado con otro tipo de sistemas cualquiera. Sólo el hardware de adquisición y de adaptación al rango sería específico para el sistema Feedback.

La razón de que las tarjetas E/S posean comunicación I2C es importante porque esta comunicación presenta una fácil integración con sistemas embebidos de pequeño coste como Arduino o Raspberry Pi y también con Simulink.

Teniendo en cuenta lo comentado en el párrafo anterior, montar en Raspberry un servicio parecido al que se expone en [9] hace que la conexión/desconexión del LRA-ULE pueda ser realizada de una forma bastante rápida, además de poder ser utilizado el sistema físico en prácticas presenciales cuando se desee.

Se presenta, por tanto, en este trabajo, una modernización de los equipos indicados utilizando sistemas embebidos de bajo coste (Arduino) que permitan la conexión/desconexión rápida de los mismos a/de la estructura de laboratorio remoto existente en la Universidad de León ([3]) y además que, durante las prácticas presenciales, los alumnos puedan realizar prácticas de una forma sencilla y con herramientas modernas.

\section{PROBLEMA Y OBJETIVOS.}

En la Universidad de León, los equipos Feedback que se utilizan se adquirieron en el año 1987 aproximadamente $\mathrm{y}$, desde entonces, han estado sirviendo a su propósito sin modernización de ningún tipo.

Evidentemente, cuando a los alumnos se les plantea utilizar equipos tan "antiguos", la primera impresión que tienen es que van a utilizar algo que no es acorde a los tiempos actuales. Hay por tanto una primera barrera de rechazo que hay que intentar eliminar.

Cuando se les indica a los alumnos que utilicen una trazadora analógica (sólo disponemos de una unidad completamente operativa) para visualizar el comportamiento de las distintas señales que se pueden adquirir, aparece otra barrera de rechazo a la utilización del equipamiento: "además de estar obsoleto, tenemos que hacer cola para utilizar el equipamiento".

Esta situación se agrava aún más cuando se utiliza alguno de los equipos Feedback dentro de la infraestructura de Laboratorio Remoto, porque si bien se aumenta el número de horas que pueden usar el equipo, en estas asignaturas introductorias al control, el caracter presencial de las prácticas debe prevalecer sobre el remoto (o al menos hay que realizar prácticas presenciales antes de las prácticas remotas), tal como se ha indicado al exponer los 7 principios de buenas prácticas educativas. 
Por lo comentado hasta ahora, es evidente que los objetivos que hay que cumplir son:

1. Modernizar los equipos Feedback de tal forma que se puedan utilizar herramientas de software para poder leer/escribir datos y realizar control sobre los mismos.

2. Que los alumnos puedan utilizar sus propios ordenadores para realizar el control sobre los equipos Feedback y, a ser posible, con tecnologías que ellos ya conozcan o les parezcan sencillas de conocer.

3. Muy importante que los alumnos puedan tener registro de datos de todas las pruebas que realicen. Así podrán reflexionar sobre los resultados en otros momentos y el uso de los equipos estará más descongestionado.

4. La modernización no puede ser cara (por necesidades presupuestarias) y debe permitir la realización de prácticas de más valor añadido: identificación de sistemas, algoritmos de control avanzados, efectos de las no linealidades en el control, comportamiento ante señales de entrada rampa, parábola o arbitrarias, efecto del periodo de muestreo en el control, efecto de los filtros de señal en el control, etc.

5. La modernización no debe ser cara también por si el alumno quisiera "montar" todo el sistema; así él podría hacerlo en su casa con un coste no superior a $70 €$; salvo, claro está, el coste del equipo Feedback. Esto posibilita dejar libertad creativa a los alumnos para que propongan prácticas o situaciones distintas y que pueden ser de gran interés.

\section{PLANTEAMIENTO.}

Con todo lo expuesto hasta ahora, la solución hardware y software que se adopta en la Universidad de León (se pretende que los alumnos la utilicen desde ahora en años posteriores) se basa en Arduíno+Simulink(Matlab) para construir un sistema de adquisición de datos que permita manipular las señales muestreadas desde Simulink (aplicando diferentes algoritmos de control) para luego actuar sobre el equipo Feedback.

Arduíno es la solución más adecuada para este fin (enfoque educativo de la solución) porque se trata de un hardware de prototipado rápido, barato y que no es ajeno para los alumnos (no tienen rechazo a utilizarlo). Se valoraron otras alternativas como Raspberry Pi, Texas Instruments C2000 Launchpad o Xilinx Zynq con ZedBoard pero si bien la primera de ellas es una buena candidata, no se conoce tanto como Arduíno y, con respecto a las otras, están más orientadas al ámbito profesional, por lo que son más complejas de utilizar.

Dentro de la solución Arduíno, se ha elegido el modelo MEGA2560 porque permite el funcionamiento en "Modo Externo" desde Simulink, por su número de entradas y salidas y su capacidad de cómputo con un precio muy ajustado.

El modelo MEGA2560 utilizado presenta carencias importantes para la adquisición de datos: no dispone de conversores digital/analógico DAC (utiliza modulación PWM para las salidas analógicas) y además los conversores analógico/digital ADC que utiliza para la lectura de señales presentan un rango de tensión en sus entradas de $0-5 \mathrm{~V}$ y 10 bits $(5 \mathrm{mV})$. Por tanto, se ha decidido utilizar/construir una etapa de adquisición de datos.

\subsection{Adquisición de datos}

Para solucionar el problema de la adquisición de datos, se ha optado por la utilización de dos módulos de adquisición comerciales: uno de salidas y otro de entradas analógicas, que realizan la comunicación con el Arduino por medio de un bus I2C. La utilización de ésta tecnología cumple dos funciones: compensar las carencias a nivel de hardware de la placa Arduino; y aislar eléctricamente la misma, minimizando el riesgo de averías que se producirían en caso de que los equipos eléctricos fallaran.

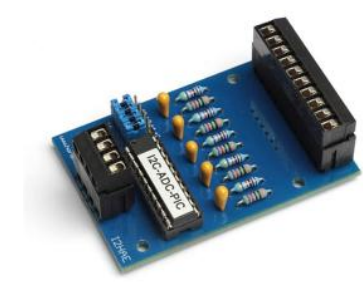

(a)

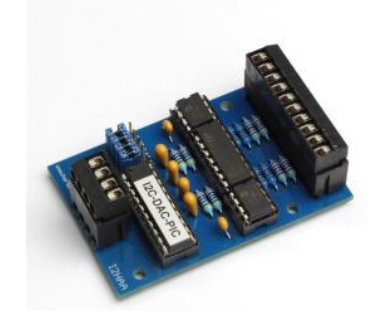

(b)
Figura 1: Móds. entradas (a) y salidas (b) analógicas.

El módulo de entradas ADC (Figura 1.a) consiste en una placa con 5 entradas analógicas con un rango de $0 \mathrm{~V}$ a $10 \mathrm{~V}$, y una resolución de 10 bits, con una frecuencia de muestreo de $80 \mathrm{~Hz}$ (suficiente para el equipo Feedback). Este módulo está controlado por un microcontrolador PIC18F13K22 y la comunicación, como se ha mencionado anteriormente, se realiza mediante I2C. Más información del mismo se puede encontrar en https://www.horter-shop.delen/i2c-din-railmodules/172-kit-i2c-analog-input-module-5-channel10-bit-4260404260745.html.

El módulo de salidas DAC (Figura 1.b) contiene 4 salidas analógicas (sustituyendo las salidas PWM del 

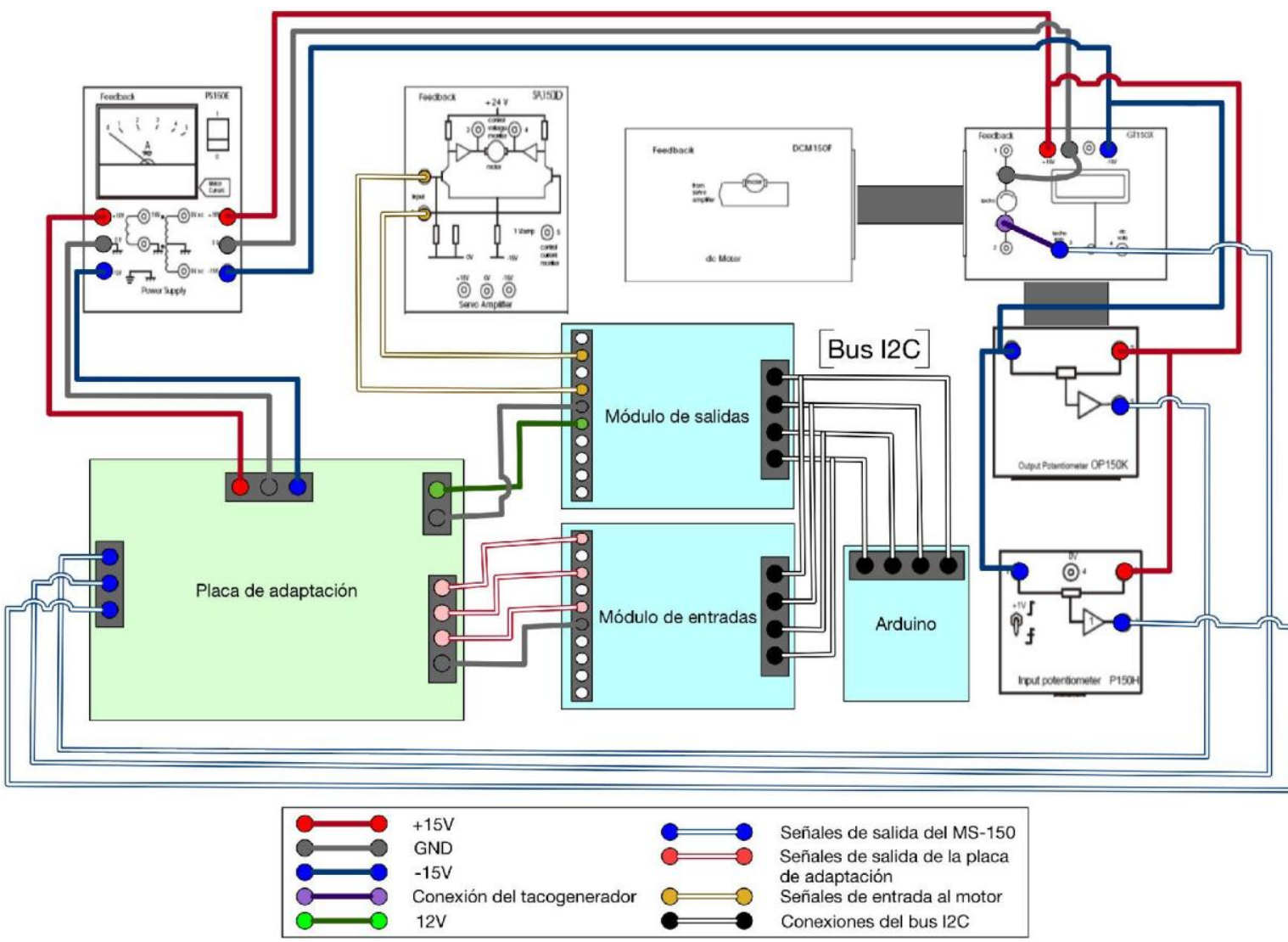

Figura 2: Conexionado elementos.

Arduino) con un rango de $0 \mathrm{~V}$ a $10 \mathrm{~V}$, y una resolución de 10 bits. Éste módulo requiere ser alimentado con una fuente de voltaje constante de 12V. Este módulo está controlado por un microcontrolador PIC18F13K22 y la comunicación, igual que en el caso anterior, se realiza mediante I2C. Más información del mismo se puede encontrar en https://www.horter-shop.de/en/home/93-kit-i2canalog-output-module-4-channel-10-bit4260404260752.html.

Por último, es necesaria la fabricación de una placa de adaptación de las señales de salida del motor, puesto que del MS-150 se obtienen unas señales cuyos rangos de valores exceden los del módulo de entradas $( \pm 15 \mathrm{~V}$ potenciómetros, $\pm 6 \mathrm{~V}$ tacómetro). En esta placa se han incorporado algunas medidas de seguridad eléctrica, puesto que los alumnos van a manipularla:

1. Protección contra inversión de polaridad en la alimentación de la placa.

2. Protección contra sobretensión de los amplificadores operacionales y contra sobretensión en las señales de entrada

3. Limitación del rango de salida a 0-10V.

\subsubsection{Comunicación.}

La placa Arduino MEGA2560 es la que se encarga de leer/escribir los datos del equipo Feedback a través de los módulos ADC y DAC mediante comunicación I2C.

El bus I2C es un tipo de bus de datos maestroesclavo en serie. La comunicación se realiza mediante 2 líneas: una señal de reloj (SCL), y una señal de datos (SDA). Ambas líneas necesitan resistencias pull-up hacia Vdd.

Los esclavos se identifican mediante direcciones de 8 bits (indicando el primero si es de lectura o escritura). La comunicación se realiza en serie, es decir, se realiza sólo con uno de los dispositivos del bus al mismo tiempo. El protocolo de comunicación para cada conexión es el siguiente:

1. El maestro inicia la transmisión, indicando si quiere leer o escribir y la dirección del esclavo.

2. Se envían los datos.

3. Se envía una señal de parada.

Para la comunicación de los módulos DAC y ADC con Arduino se utilizan las señales SDA y SCL y las señales de $5 \mathrm{~V}$ y la de masa como referencia de tensión del bus (independiente de la de la placa de adaptación).

Para leer del bus I2C los canales del módulo de entradas analógicas ADC, el fabricante indica que la sintaxis debe ser esta: 
Val(0-1023) can $1=$ ValByte $2 * 256+$ ValByte $1 ;$ Val $(0-1023)$ can $2=$ ValByte $4 * 256+$ ValByte 3 ; $\operatorname{Val}(0-1023)$ can3 $=$ ValByte6 $* 256+$ ValByte 5

Para escribir en el bus I2C, los canales del módulo de salidas analógicas DAC, el fabricante indica que deben enviarse paquetes de 3 bytes con la siguiente estructura:

Byte $0=$ Canal que se utiliza (de 0 a 3 );

Byte $1=$ Voltaje (de 0 a 1023) - Byte $2 * 256$;

Byte 2 = Voltaje (de 0 a 1023) / 256;

La comunicación del equipo Feedback con el ordenador del alumno se consigue a través de una conexión USB tipo A/B entre la placa Arduino MEGA2560 y el software Simulink (encargado de recibir, procesar, visualizar y enviar las distintas señales del equipo Feedback). La versión de Matlab utilizada ha sido la R2016b (muy importante este aspecto porque los módulos simulink encargados de la comunicación I2C no funcionaban de forma adecuada en versiones anteriores).

\subsection{Simulink}

Se considera el uso de Simulink como pieza central de software que los alumnos pueden utilizar, aunque es un programa orientado a la simulación y no está optimizado para su uso en tiempo real. La inclusión en las últimas versiones del programa de soporte para placas de prototipado rápido, además de la posibilidad de ejecución en modo externo en tiempo real solventan este problema. Es una herramienta muy visual que permite que los alumnos la utilicen sin necesidad de tener conocimientos básicos de programación.

Además, el uso de Simulink aporta las siguientes ventajas:

1. Es un software ampliamente extendido en la simulación de sistemas de control, tanto a nivel profesional como educativo, lo que reduce la curva de aprendizaje necesaria para su utilización.

2. Ofrece un gran abanico de opciones en lo referente a la simulación de sistemas de control, las cuales están automáticamente disponibles para el desarrollo de cualquier algoritmo que se desee implementar en el sistema.

3. Ofrece soporte oficial para la placa Arduino, siendo fácil e intuitiva la instalación y configuración de los paquetes de soporte.

4. Utiliza una interfaz de usuario suficientemente intuitiva como para permitir el uso de modelos creados previamente, como si de un programa con una interfaz gráfica se tratara, sin necesidad de estar familiarizado con su funcionamiento a nivel interno.

Para facilitar el uso del equipo Feedback, tanto por parte del docente como de los alumnos, se ha creado un conjunto de prácticas (ficheros en formato MDL) en los que se han incluido elementos de interacción directa como botones, ruletas, sliders, ... que pertenecen a la librería "Dashboard" del programa y se han ocultado todos aquellos bloques ajenos a las tareas de control. Así la apariencia del interfaz de usuario es similar al control teórico que se pretende estudiar en la práctica en concreto.

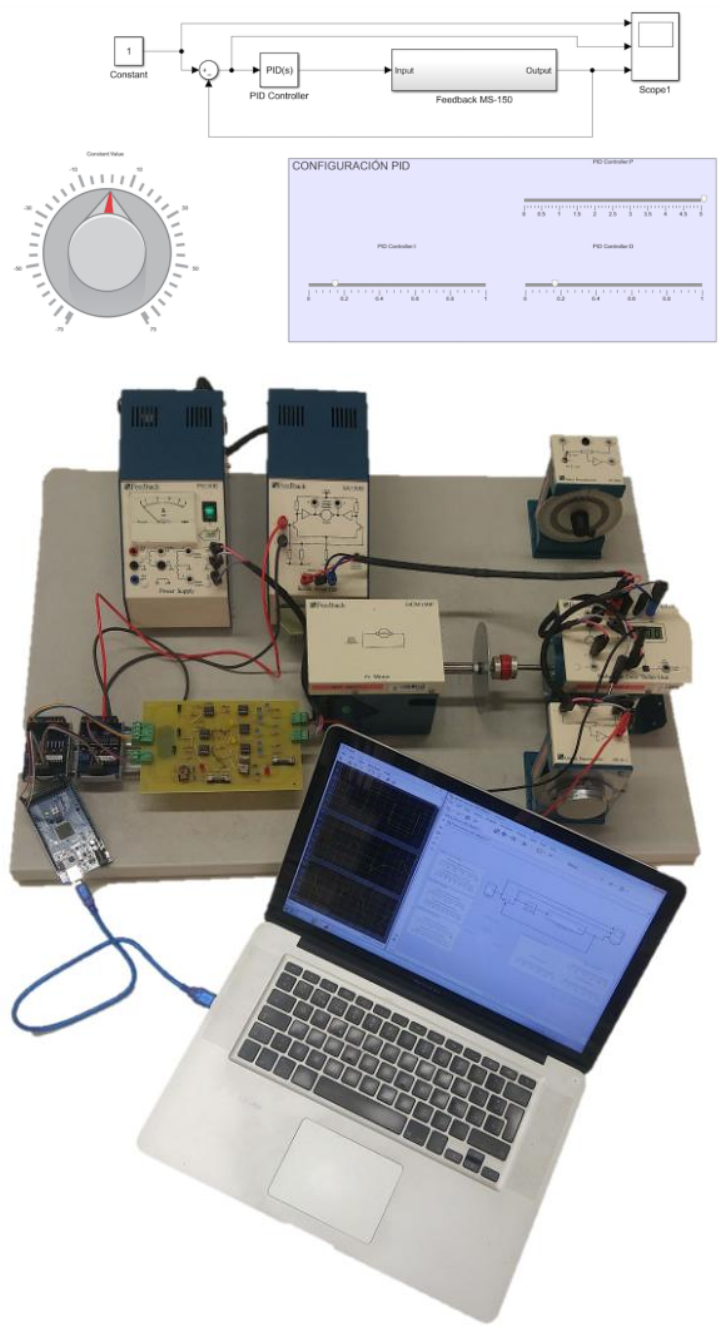

Figura 3: Interfaz usuario. Control posición PID.

Para poder comunicarse con Arduino desde Simulink es necesaria la instalación de los paquetes de soporte oficiales [7][8]. De esos paquetes se utilizan los bloques "I2C Write" y "I2C Read".

Para que el uso de los interfaces sea más flexible, se presupone que siempre se utilizará la opción de 
funcionamiento en modo externo, que supone que el alumno/profesor ejecutará el control en simulink y Arduino operará como un hardware externo dedicado sólo a la lectura/escritura de señales.

Dentro de Simulink se realiza toda la conversión y adaptación al rango de señales de valor entero a grados y rpm de tal forma que todos los bloques involucrados se han incluido dentro de un subsistema que se ha llamado "Feedback MS150". Este subsistema consta de una entrada (la del módulo servoamplificador SA150D) y 2 salidas (posición en grados del eje del motor y velocidad en rpm).

\subsubsection{Prácticas interactivas realizadas.}

En la Universidad de León se dispone de una secuencia de prácticas de control y se las ha modernizado siguiendo todos los pasos anteriores de tal forma que se dispone de un pequeño guión de cada práctica donde el alumno debe realizar una serie de tareas con el equipo físico y almacenar los datos de la sesión para luego realizar otras tareas por su cuenta fuera del laboratorio.

Las prácticas obligatorias que se proponen a los estudiantes son 10 , relativas a:

\begin{tabular}{|l|l|}
\cline { 2 - 3 } \multicolumn{1}{l|}{ P01 } & $\begin{array}{l}\text { Objetivo } \\
\text { diferentes módulos equipo Feedback. }\end{array}$ \\
\hline P02 & $\begin{array}{l}\text { Comprender operación, en cadena abierta, de } \\
\text { motor CC. accionado por servoamplificador. }\end{array}$ \\
\hline P03 & $\begin{array}{l}\text { Respuesta motor. Analizar el comportamiento } \\
\text { del motor ante cambios bruscos en su entrada. } \\
\text { Determinar la función de transferencia. }\end{array}$ \\
\hline P04 & $\begin{array}{l}\text { Señal de error. Acción de control. Señal de } \\
\text { salida. Representación de las señales y } \\
\text { características de las mismas: cte. tiempo, } \\
\text { sobreoscilación, tiempo establecimiento, .... }\end{array}$ \\
\hline P05 & $\begin{array}{l}\text { Control de posición I. Efecto de la } \\
\text { realimentación en control de posición de un } \\
\text { motor CC. }\end{array}$ \\
\hline P06 & $\begin{array}{l}\text { Control de posición II. Qué es la zona muerta } \\
\text { en un control de posición en lazo cerrado. } \\
\text { Relación con la ganancia del sistema. }\end{array}$ \\
\hline P07 & $\begin{array}{l}\text { Control de velocidad I. Entender cómo se } \\
\text { puede controlar y mantener estable la } \\
\text { velocidad de giro del motor mediante el uso } \\
\text { de un tacogenerador como realimentación. }\end{array}$ \\
\hline P08 & $\begin{array}{l}\text { Control de velocidad II. Realizar un control } \\
\text { de la velocidad de giro de un motor en los dos } \\
\text { sentidos. } \\
\text { posición en lazo cerrado. } \\
\text { control de posición III. Implementar un } \\
\text { lantrol doblemente realimentado que mejora } \\
\text { utilizar bloques no lineales incluidos en }\end{array}$ \\
\hline
\end{tabular}

Simulink, tanto como reguladores, como integrantes de otros elementos.

\section{RESULTADOS-CONCLUSIONES.}

A fecha de hoy (hemos acabado en estos días la implementación de la solución), sólo hemos podido evaluar el comportamiento del hardware y las posibilidades de integración que ofrece. Pretendemos en el curso que viene hacer uso de la solución con alumnos de los primeros cursos de Ingeniería de Control. No obstante, a pesar de sólo ofrecer el punto de vista del docente, la modernización del equipo Feedback ofrece:

1. El docente puede ofrecer otro tipo de prácticas más avanzadas utilizando el equipo Feedback, desde el momento que la estructura se sustenta en realizar el control de un sistema muestreado.

2. El cableado de la solución se simplifica enormemente y se parece más a los lazos de control que pueden encontrarse en la industria.

3. La capacidad de muestreo de las tarjetas utilizadas $(80 \mathrm{~Hz})$ es más que suficiente para la dinámica del sistema.

4. La estructura propuesta es muy flexible en el sentido de que se pueden visualizar muchos efectos: cómo afecta el tiempo de muestreo al control, aparición de ciclos límite con controles no lineales, cómo afecta la ganancia de un controlador al control y a las características del ciclo límite, cuál es el efecto de la zona muerta, cuál es el efecto de la saturación, cuál es el efecto de la histéresis y cómo evoluciona el sistema cuando se introduce un regulador cualquiera o el comportamiento en frecuencia del mismo.

5. Los alumnos podrán obtener todas las muestras de la sesión de prácticas y ya dentro del workspace de Matlab, sin perder tiempo en conversiones de ficheros $o$ enlaces a fuentes de datos remotas.

6. Los alumnos visualizan una gráfica de evolución de la señal de salida al mismo tiempo que observan la evolución del motor.

7. El coste del hardware que se ha tenido que realizar no supera los 100€; coste mínimo desde el momento que la vida del equipo Feedback se alarga y además se puede utilizar para explicar otros conceptos para los que no fue diseñado inicialmente.

8. Los interfaces de usuario creados son muy intuitivos y la operación de la estructura es 
muy estable, sin ningún problema de operación.

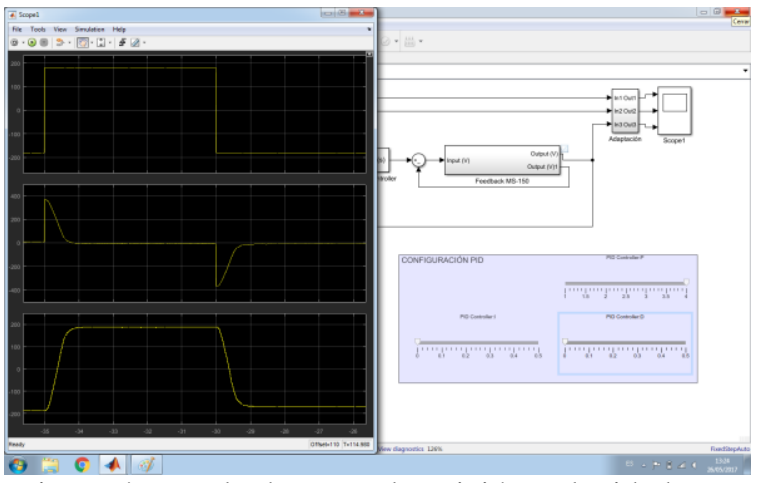

Figura 4: Resultado control posición-velocidad PID (sólo acción proporcional $\mathrm{Kp}=4$ ).

\section{Agradecimientos}

Este trabajo ha sido desarrollado por REMOTLAB, Grupo de Innovación Docente de la Universidad de León (GID18), y financiado en parte por la convocatoria PAGID2016 de dicha universidad.

\section{Referencias}

[1] A.W. Chickering, and S.C. Ehrmann, "Implementing the Seven Principles of Good Practice in Undergraduate Education: Technology as Lever," American Association for Higher Education Bulletin, California, USA, 1996.

[2] A.W. Chickering, and Z.F. Gansom, "Applying the Seven Principles for Good Practice in Undergraduate Education: New directions for Teaching and Learning" (Chickering, A. W. \& Gamson, Z. F.), 47, Jossey-Bass, San Francisco, CA, USA, 1991.

[3] M. Domínguez, P. Reguera, and J.J. Fuertes, "Laboratorio remoto para la enseñanza de la Automática en la Universidad de León”, RIAI, Madrid, Spain, 2005 (In Spanish).

[4] Domínguez, M., Marcos, D., Reguera, P., González, J.J., Blazquez, L.F., (2001) "Connection Pilot Plant to the Internet", IFAC Internet Based Control Education. IBCE01, Madrid. España.

[5] Dormido,S. (2002). "Control Learning: present and future". 15th IFAC World Congress. Barcelona.

[6] R.M. Felder, G.N. Felder, and E.J. Dietz, "A Longitudinal Study of Engineering Student Performance and Retention Vs Comparisons with Traditionally Taught Students," Journal of Engineering Education, vol. 87, n 4, pp. 469480, 1998.

[7] Mathworks (2017). Arduino Support from MATLAB. https://es.mathworks.com/hardwaresupport/arduino-matlab.html

[8] Mathworks (2017). Arduino Support from Simulink. https://es.mathworks.com/hardwaresupport/arduino-simulink.html

[9] Reguera, P., García, D., Domínguez, M., Prada, M.A., Alonso, S., (2015) "A Low-Cost open source hardware in Control Education. Case study: Arduino-Feedback MS-150". Science Direct, IFAC-PapersOnLine 48-29 (2015) 117122 